\title{
BMJ Open Are adverse childhood experiences associated with late-life cognitive performance across racial/ethnic groups: results from the Kaiser Healthy Aging and Diverse Life Experiences study baseline
}

Audra L Gold, ${ }^{1}$ Erika Meza, ${ }^{1}$ Sarah F Ackley, ${ }^{1}$ Dan M Mungas, ${ }^{2}$ Rachel A Whitmer, ${ }^{2,3}$ Elizabeth Rose Mayeda, ${ }^{4}$ Sunita Miles, ${ }^{3}$ Chloe W Eng, ${ }^{1}$ Paola Gilsanz (D) , ${ }^{3}$ M Maria Glymour (D) ${ }^{1}$

To cite: Gold AL, Meza E, Ackley SF, et al. Are adverse childhood experiences associated with late-life cognitive performance across racial/ethnic groups: results from the Kaiser Healthy Aging and Diverse Life Experiences study baseline. BMJ Open 2021;11:e042125. doi:10.1136/ bmjopen-2020-042125

- Prepublication history and additional materials for this paper is available online. To view these files, please visit the journal online (http://dx.doi org/10.1136/bmjopen-2020042125).

Received 30 June 2020 Revised 23 November 2020 Accepted 10 December 2020

Check for updates

(c) Author(s) (or their employer(s)) 2021. Re-use permitted under CC BY-NC. No commercial re-use. See rights and permissions. Published by BMJ.

For numbered affiliations see end of article.

Correspondence to Dr M Maria Glymour; Maria.glymour@ucsf.edu

\section{ABSTRACT}

Objectives Evidence on adverse childhood experiences (ACEs) and late-life cognitive outcomes is inconsistent, with little research among diverse racial/ethnic groups. We investigated whether ACE exposures were associated with worse late-life cognition for all racial/ethnic groups and at different ages of exposure.

Design Covariate-adjusted mixed-effects linear regression models estimated associations of: (1) total number of ACEs experienced, (2) earliest age when ACE occurred and (3) type of ACE with overall cognition. Setting Kaiser Permanente Northern California members aged 65 years and older, living in Northern California.

Participants Kaiser Healthy Aging and Diverse Life Experiences study baseline participants, aged 65 years and older ( $n=1661$; including 403 Asian-American, 338 Latino, 427 Black and 493 white participants).

Results Most respondents (69\%) reported one or more ACE, most frequently family illness (36\%), domestic violence (23\%) and parental divorce (22\%). ACE count was not adversely associated with cognition overall $(\beta=0.01$; $95 \% \mathrm{Cl}-0.01$ to 0.03 ), in any racial/ethnic group or for any age category of exposure. Pooling across all race/ ethnicities, parent's remarriage $(\beta=-0.11 ; 95 \% \mathrm{Cl}-0.20$ to -0.03$)$, mother's death $(\beta=-0.18 ; 95 \% \mathrm{Cl}-0.30$ to $-0.07)$ and father's death $(\beta=-0.11 ; 95 \% \mathrm{Cl}-0.20$ to -0.01 ) were associated with worse cognition.

Conclusion Adverse childhood exposures overall were not associated with worse cognition in older adults in a diverse sample, although three ACEs were associated with worse cognitive outcomes.

\section{INTRODUCTION}

Adverse childhood experiences (ACEs), such as abuse, violence and household dysfunction have lasting harmful impacts on adult physical and mental health, ${ }^{1-3}$ but evidence on the effect of ACEs on late-life cognitive performance and decline is mixed. ${ }^{4-6}$

\section{Strengths and limitations of this study}

- Evidence on the effect of adverse childhood experiences (ACEs) on late-life cognitive performance and decline is mixed, with very little research conducted in populations with substantial racial/ethnic diversity.

- In the Kaiser Healthy Aging and Diverse Life Experiences cohort, comprising participants aged 65 years and older identifying as Black, AsianAmerican, Latino or white, exposure to ACEs was not associated with worse late-life cognition in any racial/ethnic group, and associations did not differ by age of ACE exposure.

- Only parental remarriage and parental death were consistently associated with worse cognitive outcomes in late life.

- Primary limitations of the present study include reliance on a cross-sectional sample and self-reported ACEs.

Understanding the links between ACE exposure and late-life cognitive function is critical because low cognitive function, especially memory, is a strong predictor of risk of dementia, mortality, institutionalisation, self-rated health and disability, among other health outcomes. ${ }^{1}{ }^{7-9}$ Prior studies indicate heterogeneities in the association of ACEs and cognitive outcomes by age of exposure, type of ACE, race/ethnicity and sources of resilience. For example, Ravona-Springer et $a l^{10}$ found that death of a parent during childhood was associated with substantially higher risk of dementia when the experience occurred between the ages of 0 and 6 years, but the excess dementia risk attenuated 
the older the age of ACE exposure. Additional findings suggest that while some ACEs appear to adversely affect late-life cognitive functioning, other ACEs predict better cognitive outcomes. ${ }^{6} 10$

Both exposure to and consequences of ACEs may differ by race/ethnicity. Significant racial and ethnic differences in the prevalence of ACEs as well as between types of adversities have been documented. ${ }^{11}$ To date, there has been only one multiracial study evaluating ACEs and cognition: Barnes et at found no association between early-life adverse events and cognitive decline in whites, while early-life food deprivation was associated with better cognitive outcomes for African Americans. ${ }^{4}$ No other studies have directly compared effects across racial/ ethnic groups.

We investigated the association of ACEs with laterlife cognitive performance in the Kaiser Healthy Aging and Diverse Life Experiences (KHANDLE) cohort. We hypothesised that ACE exposure would predict worse late-life cognition for all racial/ethnic groups, with the largest effects associated with experiences when aged 0-6 years.

\section{METHODS}

\section{Study participants and data collection}

We used baseline data from the KHANDLE cohort, which comprises community-dwelling older adults residing in the San Francisco Bay and Sacramento areas of California. KHANDLE aims to evaluate how race/ethnicity and life course health and sociocultural factors influence late-life brain health and cognitive decline. Individuals eligible for KHANDLE: were long-term members of Kaiser Permanente Northern California (KPNC), an integrated healthcare delivery system; were aged 65 years or older on 1 January 2017; spoke English or Spanish; and had previously participated in Kaiser Permanente multiphasic health check-up exams between 1964 and 1985. Stratified random sampling by race/ethnicity and educational attainment was used with the goal of recruiting approximately equal proportions of Asian, Black, Latino and white participants and achieving diversity in educational attainment. Exclusion criteria included: electronic medical record diagnosis of dementia or other neurodegenerative disease (frontotemporal dementia, Lewy body disease, Pick's disease, Parkinson's disease with dementia and Huntington's disease) and presence of health conditions that would impede participation in study interviews, defined by hospice activity in the past 12 months, history of severe chronic obstructive pulmonary disease in the past 6 months, congestive heart failure hospitalisations in the past 6 months and history of end-stage renal disease or dialysis in the past 12 months. At baseline, 1712 individuals were enrolled. Individuals who self-identified as Native Americans $(n=3)$ or refused to self-identify race/ ethnicity $(n=1)$ were dropped from the sample used in this analysis. After excluding 13 individuals who were missing all cognitive measures used in this analysis and 34 individuals missing all ACEs, the final analytic sample size was 1661. All respondents provided informed consent and completed an interview in English or Spanish either in-home or at a Kaiser Permanente Facility (approximately $40 \%$ of baseline interviews were conducted at a facility and $60 \%$ in-home).

KPNC is a large, integrated healthcare delivery system that provides comprehensive medical care to over 4 million members. ${ }^{12}$ Prior work indicated the member population was generally representative of the overall regional population, though individuals at extreme tails of the income distribution were under-represented. ${ }^{13-15}$ The KPNC older adult population (aged 65+ years) are generally similar to the population of seniors residing in Northern California with respect to medical history of chronic conditions, including diabetes, hypertension, heart disease and asthma, and lifestyle factors, including smoking, obesity and sedentary lifestyle. ${ }^{15}$

\section{Measures}

Our cognitive outcomes are standardised scores from the following three cognitive domains: verbal episodic memory, semantic memory and executive functioning. These scores were obtained from the Spanish and English Neuropsychological Assessment Scales (SENAS), which was given to all participants in their preferred language (English or Spanish). ${ }^{16}$ The SENAS is a battery of cognitive tests that has previously undergone extensive development for valid comparisons of cognition across racial/ ethnic and linguistically diverse groups. Verbal episodic memory composite scores were derived from a multiracial word-list learning test. Semantic memory composite scores were derived from verbal (object naming) and non-verbal (picture association) tests. Executive function composite scores were obtained using component tasks of category fluency, phonemic (letter) fluency and working memory (digit-span backward, visual-span backward and list sorting). Details of the administration procedures, development and psychometric characteristics have been extensively described in previous publications. ${ }^{16}$ Analyses used cognitive data for everyone who had cognitive measures for at least one of the three cognitive domains.

KHANDLE fielded a modified version of the assessment of ACEs used in the Reasons for Geographic and Racial Disparities in Stroke cohort. ${ }^{17}$ Participants were asked aloud by the interviewer if they had experienced each of nine ACEs when they were age 16 years or younger: parents were divorced or separated; parents remarried; witnessed domestic violence; substance abuse by a family member; loss of a job by a parent; parent had to go to jail; serious illness of a family member; death of mother; and death of father. If any ACE was experienced, respondents were asked the youngest age at which they experienced the event. A composite ACE score was constructed as a count of the number ever experienced, with the scores ranging from 0 if no ACE had been experienced to 9 if every ACE had been experienced. For individuals missing one or more ACE item, we imputed the values to the 
average of the total observed ACEs for that individual (ie, if the individual responded to six ACE items and endorsed three of them, the values of the missing three items were imputed to 0.5$)$. Since few people reported more than four ACEs $(n=161)$, the count of ACEs was top coded at 4 for our analyses. Age-specific ACEs were constructed as the count of the number of experiences reported as first occurring in specific age categories $(0-6,7-12$ and $13-16$ years).

Because ACEs are experienced in early life, there are few plausible confounders that might influence both ACEs and late-life cognitive outcomes. All models were either adjusted for or stratified by race/ethnicity (classified as Black, white, Latino or Asian). All models were also adjusted for linear and quadratic terms for year of age over 65 years at cognitive assessment, sex and parental education. Parental educational attainment was reported by the respondent as highest level of education completed. Maternal and paternal education for primary and secondary education was coded as number of years of primary or secondary education, ranging from 0 to 12 . We additionally adjusted for parental higher education as a continuous predictor: $0=$ no higher education; $1=$ some college but no degree or associates degree; 2=bachelor's degree, master's degree or other higher education. If values for parental education were missing $(\mathrm{n}=276$ and $\mathrm{n}=417$ missing for maternal and paternal, respectively), they were coded as 0 (lowest category), and we additionally adjusted for an indicator variable for missingness.

\section{Statistical analysis}

Baseline variables gender, race/ethnicity and parental education were tabulated by ACE composite score. The prevalence of each ACE was estimated for the entire sample and stratified by race/ethnicity.

If the effect of ACEs were the same for all three domains of cognition, it would be most efficient to estimate a single mixed model including each individual's three outcome assessments (verbal memory, semantic memory and executive function) and derive a single effect estimate applicable to all domains. This added efficiency is especially important when estimating race/ethnicity specific effects where sample sizes are smaller. Before estimating such a model, we first had to assess whether it was appropriate to estimate a single effect of ACE exposure on all three cognitive domains within each racial/ethnic group.

In initial models, we therefore tested for domainspecific effects of composite ACE score on cognition for each racial/ethnic group. To do this, we used a mixedeffects linear regression model with the three standardised cognitive domains as outcomes with random intercepts to account for within-person correlation between cognitive domains. All models were controlled for indicators of cognitive domain (eg, verbal memory or semantic memory, with executive function treated as the reference outcome), allowing for the possibility that average score differs between domains. We also included interactions between race/ethnicity and domain (allowing for the possibility that domain differences vary by race/ethnicity) and interactions between each person's composite ACE score and each race/ethnicity-domain combination (allowing for the possibility that the effect of ACE exposure differs for any combination of race/ethnicity and cognitive domain). An F-test for the null hypothesis that the race/ethnicity-specific ACE associations with cognition varied significantly across domains indicated evidence of heterogeneity $(\mathrm{p}=0.09)$. When we evaluated individual comparisons, we found one significant domainspecific difference: the association between ACE score and semantic memory among Asian American respondents. For both other domains in Asian Americans and for all domains in the other three racial/ethnic groups, the statistical tests indicated estimation of a single parameter for estimated effects of ACEs on cognition was appropriate. In all subsequent models, interactions between Asian American, semantic memory and the ACE measure were included to estimate the association of ACEs with semantic memory among Asian Americans separately from all other associations. In these models, the coefficient for the ACE measure can be interpreted as the association of ACEs with cognition, averaged across domains (results are very similar to what would be obtained if the different domains were averaged in advance and treated as a single outcome) except excluding the effect of ACEs on semantic memory among Asian Americans. Associations between ACE exposure and semantic memory among Asian American respondents are reported in online supplemental appendix tables 1-4.

We then used covariate-adjusted linear mixed models to estimate the association of composite ACE score with cognition in pooled analyses and in models stratified by race-ethnicity. To evaluate whether differences in coefficients between racial/ethnic groups were statistically significant, we incorporated race/ethnicity by ACE score interactions and used an F-test. Because of limited prior evidence on racial/ethnic specific associations, we present these even when the F-test indicated no evidence of statistically significant heterogeneity.

Covariate adjusted mixed-effects linear regression models were also estimated to evaluate the association between composite ACE scores at specific age categories (0-6, 7-12 and 13-16 years) and cognition for the full sample and in models stratified by race/ethnicity.

Finally, we evaluated the association of each of the nine individual ACEs and standardised cognitive score and whether these associations differed by race/ethnicity or by age ranges of exposure $(0-6 ; 7-12 ; 13-16$ years). Results for age-specific associations with individual ACE exposures are given in the online supplemental appendix table 5 .

Analyses were conducted using STATA SE V.15.

\section{RESULTS}

Among the 1661 participants enrolled in the KHANDLE baseline, $69 \%$ reported experiencing at least one ACE 
Table 1 Descriptive statistics, KHANDLE cohort

\begin{tabular}{|c|c|c|c|c|c|c|}
\hline \multirow[b]{2}{*}{ Variable } & \multicolumn{5}{|c|}{ Composite ACE score } & \multirow[b]{2}{*}{$4+$} \\
\hline & Level & 0 & 1 & 2 & 3 & \\
\hline $\mathrm{N},(\%)$ & 1661 & $513(100)$ & $448(100)$ & $336(100)$ & $203(100)$ & $161(100)$ \\
\hline \multirow[t]{2}{*}{ Gender, n (\%) } & Female & $223(43.5)$ & $182(40.6)$ & $133(39.6)$ & $74(36.5)$ & $63(39.1)$ \\
\hline & Male & $290(56.5)$ & $266(59.4)$ & $203(60.4)$ & $129(63.5)$ & 98 (60.9) \\
\hline \multirow{3}{*}{ Race/ethnicity, n (\%) } & Black & $105(20.5)$ & $116(25.9)$ & $92(27.4)$ & $66(32.5)$ & $48(29.8)$ \\
\hline & Latino & $71(13.8)$ & $82(18.3)$ & $85(25.3)$ & $51(25.1)$ & 49 (30.4) \\
\hline & White & $156(30.4)$ & $139(31.0)$ & $91(27.1)$ & $57(28.1)$ & $50(31.1)$ \\
\hline \multirow[t]{2}{*}{ Maternal education } & $\begin{array}{l}\text { Mean years of education } \\
\text { among those with } \leq 12 \text { years, } \\
\text { mean (SD) }\end{array}$ & $8.46(4.73)$ & $7.76(4.87)$ & $7.78(4.74)$ & $7.98(5.00)$ & $7.83(4.76)$ \\
\hline & Bachelor's or more, $\mathrm{n}(\%)$ & 63 (12.3) & $34(7.6)$ & $32(9.5)$ & $18(8.9)$ & $10(6.2)$ \\
\hline \multirow[t]{3}{*}{ Paternal education } & $\begin{array}{l}\text { Mean years of education } \\
\text { among those with } \leq 12 \text { years, } \\
\text { mean (SD) }\end{array}$ & $8.51(4.75)$ & $7.29(5.16)$ & $6.82(5.36)$ & $6.19(5.21)$ & $6.53(5.45)$ \\
\hline & $\begin{array}{l}\text { Some college but no degree/ } \\
\text { associates degree, } \mathrm{n}(\%)\end{array}$ & $60(11.7)$ & $43(9.6)$ & $32(9.5)$ & $21(10.3)$ & $12(7.5)$ \\
\hline & Bachelor's or more, n (\%) & $110(21.4)$ & $70(15.6)$ & $46(13.7)$ & $14(6.9)$ & 15 (9.3) \\
\hline
\end{tabular}

ACE, adverse childhood experience; KHANDLE, Kaiser Healthy Aging and Diverse Life Experiences.

prior to age 16 years: 448 individuals $(27 \%)$ reported experiencing 1 ACE; 336 (20\%) reported 2 ACEs; 203 $(12 \%)$ reported 3 ACEs and $161(10 \%)$ reported $4+$ ACEs (table 1). Among the nine individual ACEs, illness in the family had the highest overall prevalence at $36 \%$, followed by domestic violence (23\%) and parental divorce (22\%) (figure 1). Both the distribution of total ACE score and the prevalence of each specific ACE varied by race/ ethnicity.
In covariate-adjusted mixed-effects linear regression models, there was no association between the composite ACE score and standardised cognition when considering all racial/ethnic groups in a pooled analysis $(\beta=0.01$; $95 \%$ CI -0.01 to 0.03 ) (table 2). Age-specific ACE exposures were not significantly associated with cognition (ACE scores ages $0-6, \beta=0.01 ; 95 \%$ CI -0.03 to 0.05 ; ACE scores ages $7-12, \beta=-0.03 ; 95 \% \mathrm{CI}-0.07$ to 0.01 ; or ACE score ages $13-16, \beta=0.01 ; 95 \%$ CI -0.05 to 0.06 ) (table 2 ).

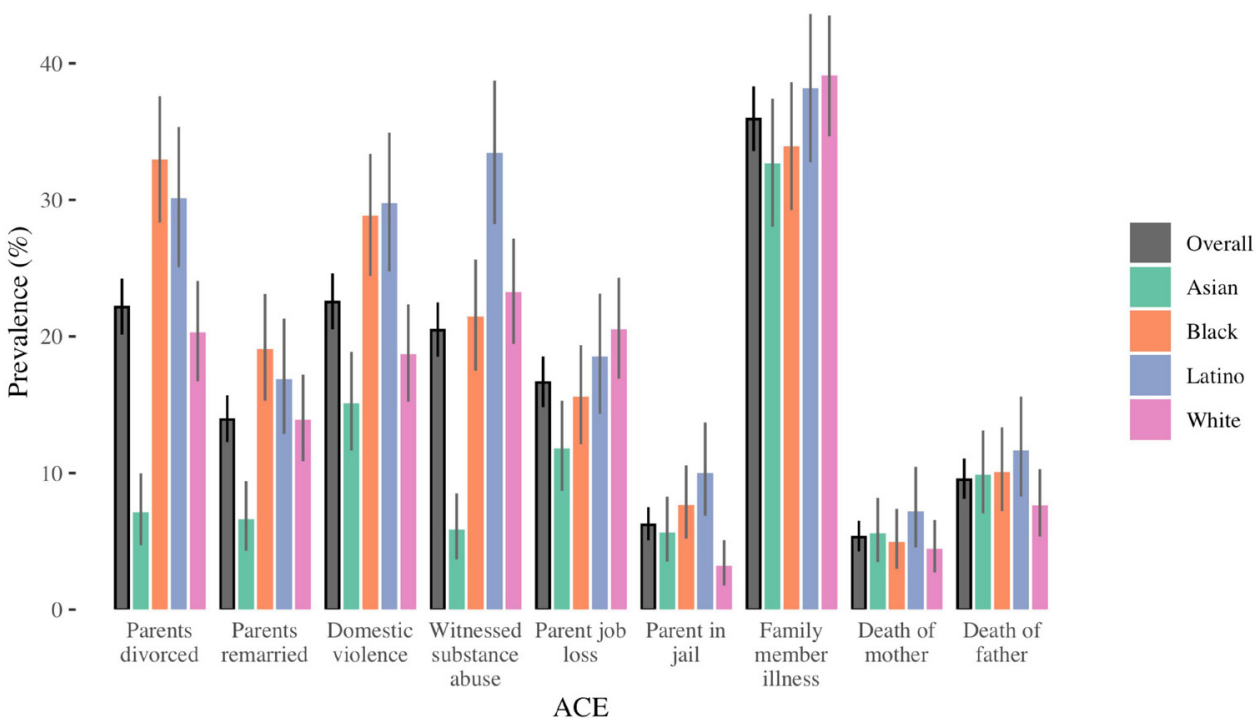

Figure 1 Grey vertical line segments indicate exact binomial Cls. 
Table 2 Mixed-effects linear regression coefficients (95\% Cls) for the difference in cognitive scores associated with total childhood or age-specific count of ACEs (0 to 4+ years), overall and stratified by race/ethnicity

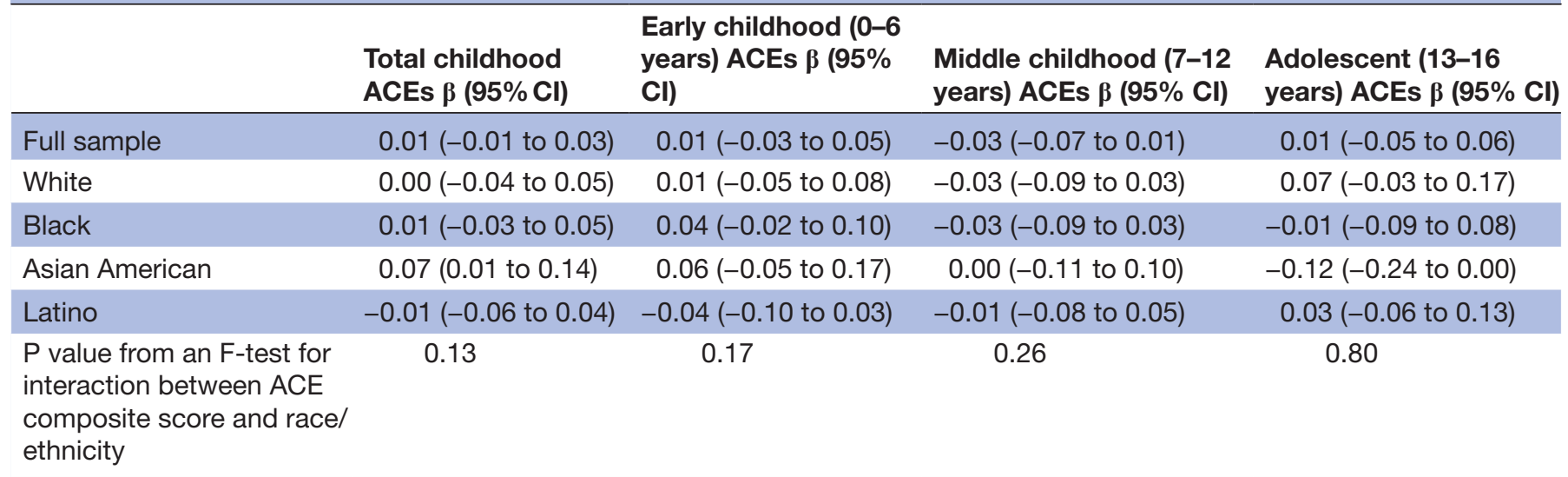

*All models adjusted for age (linear and quadratic), sex, parental education and race/ethnicity (unless stratified by race/ethnicity). †All models provide a single coefficient for associations with verbal episodic memory, semantic memory and executive function, with the exception of the coefficients for ACE association with semantic memory among Asian Americans, which are estimated separately and presented in the supplement.

ACEs, adverse childhood experiences.

When evaluating the association between total ACE score and cognition for each racial/ethnic group, the only apparent association was for Asian Americans, among whom each unit increase in the composite ACE was associated with better cognitive scores (estimated association based on verbal memory and executive function, $\beta=0.07$; $95 \%$ CI 0.01 to 0.14 ). Although this individual racespecific association was statistically significant, the overall test for differences in the association of ACE composite score and cognition across racial/ethnic groups was not significant $(\mathrm{p}=0.13)$ after excluding the single Asian semantic memory comparison.

Interactions between race/ethnicity and age-specific ACE exposures were also non-significant. There was no evidence of associations between age-specific ACE exposures and cognition overall, although Asian Americans exposed to ACEs age 13-16 years averaged worse cognition $\beta=-0.12 ; 95 \%$ CI -0.24 to 0.00 ) (table 2 ).

Pooling across all race/ethnicities, three types of ACE exposures were associated with worse cognition: parent remarried; death of mother; and death of a father (table 3). Although there were some differences in the associations of individual ACEs with standardised cognition between racial/ethnic groups, differences were consistent with chance (ie, the tests of heterogeneity in the ACE associations with cognition across racial/ethnic groups were not statistically significant). Patterns were generally similar when evaluating cognition based on indicator variables for age of first exposure overall and race/ethnicity (online supplemental appendix table 1 ).

\section{DISCUSSION}

Retrospectively reported exposure to childhood ACEs was prevalent in a sample of long-term elderly KPNC members, but ACEs were not associated with cognition in later life among white, Black or Latino respondents. Results were similar for total ACE count and ACE exposures during age groups categorised as $0-6,7-12$ and 13-16 years. Among Asian American respondents, higher ACE count was associated with slightly better overall cognition, although a difference too small to be of notable clinical significance. Among the individual ACEs, three experiences were associated with significantly worse cognition when pooling across all racial/ethnic groups, but when examining each racial/ethnic group separately, point estimates indicated adverse associations only for parents remarried and death of a mother (although with wide CIs in racial/ethnic group specific estimates). For other ACEs, associations were inconsistent and, in several instances, positive. All racial/ethnic differences in ACE by cognition associations were consistent with chance.

Our finding of no association between overall ACE count and cognition is surprising in light of prior evidence that ACEs influence multiple domains of adult physical health. ${ }^{118}$ Early life stress predicts both hippocampus and amygdala development in children as well as children's cognitive and affective functioning. ${ }^{19-21}$ However, children's responses to such adversity are very heterogeneous, and both social and genetic factors may ameliorate or outweigh the effects of adversity as a child matures. ${ }^{22}$ However, prior findings in early work on ACEs and cognition has been mixed and has been conducted in predominantly white samples. Very few prior studies include multiracial samples or assessments with both agespecific ACE exposure and late-life cognitive outcomes. This is important to evaluate because race/ethnicity is strongly associated with economic, social, political and environmental factors that influence cognitive ageing. These factors may modify the consequences of ACEs for cognition in late life or may create selection processes 
Table 3 Mixed effects linear regression coefficients (95\% Cls) for the difference in memory scores associated with each ACE, overall and stratified by race/ethnicity

\begin{tabular}{|c|c|c|c|c|c|c|}
\hline ACEs & Overall $\beta(95 \% \mathrm{Cl})$ & $\begin{array}{l}\text { White } \beta \\
(95 \% \mathrm{Cl})\end{array}$ & $\begin{array}{l}\text { Black } \beta \\
(95 \% \mathrm{Cl})\end{array}$ & $\begin{array}{l}\text { Asian } \beta \\
(95 \% \mathrm{Cl})\end{array}$ & Latino $\beta(95 \% \mathrm{Cl})$ & $\begin{array}{l}\text { interaction } \\
\text { with race/ } \\
\text { ethnicity* }\end{array}$ \\
\hline \multirow{2}{*}{ Parents divorced } & -0.03 & -0.10 & -0.03 & 0.09 & -0.00 & 0.20 \\
\hline & $(-0.11$ to 0.04$)$ & $(-0.24$ to 0.04$)$ & $(-0.15$ to 0.09$)$ & $(-0.16$ to 0.35$)$ & $(-0.14$ to 0.14$)$ & \\
\hline \multirow[t]{2}{*}{ Parents remarried } & -0.11 & -0.10 & -0.04 & -0.08 & -0.18 & 0.74 \\
\hline & $(-0.20$ to -0.03$)$ & $(-0.26$ to 0.07$)$ & $(-0.18$ to 0.09$)$ & $(-0.34$ to 0.19$)$ & $(-0.34$ to -0.03$)$ & \\
\hline \multirow[t]{2}{*}{ Domestic violence } & 0.01 & 0.07 & -0.00 & 0.19 & -0.07 & 0.11 \\
\hline & $(-0.06$ to 0.08$)$ & $(-0.07$ to 0.21$)$ & $(-0.12$ to 0.12$)$ & (0.00 to 0.38$)$ & $(-0.21$ to 0.06$)$ & \\
\hline \multirow{2}{*}{$\begin{array}{l}\text { Witnessed substance } \\
\text { abuse }\end{array}$} & -0.06 & -0.08 & 0.02 & 0.18 & -0.16 & 0.05 \\
\hline & $(-0.14$ to 0.01$)$ & $(-0.21$ to 0.06$)$ & $(-0.11$ to 0.15$)$ & $(-0.10$ to 0.46$)$ & $(-0.29$ to -0.02$)$ & \\
\hline \multirow[t]{2}{*}{ Parent job loss } & 0.04 & 0.10 & -0.04 & 0.16 & -0.04 & 0.26 \\
\hline & $(-0.04$ to 0.11$)$ & (-0.04 to 0.23$)$ & $(-0.19$ to 0.10$)$ & $(-0.04$ to 0.37$)$ & $(-0.19$ to 0.12$)$ & \\
\hline \multirow[t]{2}{*}{ Parent in jail } & -0.10 & -0.16 & -0.09 & 0.17 & -0.13 & 0.29 \\
\hline & $(-0.21$ to 0.01$)$ & $(-0.43$ to 0.10$)$ & $(-0.27$ to 0.08$)$ & $(-0.11$ to 0.45$)$ & $(-0.32$ to 0.05$)$ & \\
\hline \multirow[t]{2}{*}{ Family member illness } & 0.02 & 0.06 & -0.05 & 0.11 & 0.00 & 0.29 \\
\hline & $(-0.04$ to 0.08$)$ & $(-0.06$ to 0.17$)$ & $(-0.17$ to 0.06$)$ & $(-0.04$ to 0.26$)$ & $(-0.12$ to 0.13$)$ & \\
\hline \multirow[t]{2}{*}{ Death of mother } & -0.18 & -0.10 & -0.15 & -0.19 & -0.29 & 0.67 \\
\hline & $(-0.30$ to -0.07$)$ & $(-0.34$ to 0.14$)$ & $(-0.36$ to 0.06$)$ & $(-0.48$ to 0.10$)$ & $(-0.49$ to -0.09$)$ & \\
\hline \multirow[t]{2}{*}{ Death of father } & -0.11 & 0.05 & -0.14 & -0.10 & -0.21 & 0.55 \\
\hline & $(-0.20$ to -0.01$)$ & $(-0.15$ to 0.25$)$ & $(-0.31$ to 0.03$)$ & $(-0.33$ to 0.12$)$ & $(-0.39$ to -0.02$)$ & \\
\hline
\end{tabular}

${ }^{*} \mathrm{P}$ values reflect the $\mathrm{F}$-test of the interaction between individual $A C E s$ and race/ethnicity.

†All models adjusted for age (linear and quadratic), sex, parental education and race/ethnicity (unless stratified by race/ethnicity).

$\ddagger$ All models provide a single coefficient for associations with verbal episodic memory, semantic memory and executive function, with the exception that coefficients for ACE association with semantic memory among Asian Americans are estimated separately and presented in the supplement.

ACEs, adverse childhood experiences.

such that only especially resilient individuals survive to late life. For example, increased exposure to extreme adversity across the lifecourse may blunt the special relevance of childhood adversity among racial/ethnic minorities.

Our findings do suggest that parental remarriage and parental death are associated with worse cognitive outcomes in late life. The exposures were common, especially for racial/ethnic minorities. The importance of these experiences over other adversities may imply the special relevance of a child being separated from the parent.

Prior studies have documented significant racial and ethnic differences in the prevalence of total ACEs as well as between types of adversity, such as incarceration of a family member versus domestic violence, in the USA. ${ }^{11}$ ACEs are strongly patterned by socioeconomic status and neighbourhood context. ${ }^{23}$ Barnes et at reported that in a cohort of 6105 older African American and white individuals followed for up to 16 years, there was no association between early life adverse events and rate of cognitive decline in whites, while food deprivation and being thinner than average in early life were associated with better cognitive outcomes for African American individuals. ${ }^{4}$ Food deprivation is not commonly used in ACE surveys and was not included in the KHANDLE baseline survey.

Although point estimates for the effects of ACEs among whites were generally more adverse than for other racial/ ethnic groups, only for Asian American did we find evidence of a statistically significant difference. Among Asian Americans, higher ACE count was associated with better overall cognition. It is difficult to theorise how ACEs might enhance later life cognition. The positive association observed among older Asian Americans may be due to chance, selective survival or differential recall. Especially among Asian American participants in KHANDLE, many of whom were born outside the USA, individuals who have migrated, survived to late life and enrolled in the study may be an extraordinarily resilient group. This type of selection could lead to inverse associations if both ACE exposure and other determinants of late-life cognition influence the process that leads to study enrolment. Chance is also a plausible explanation, as indicated by the 
non-significant F-test for heterogeneity between racial/ ethnic groups.

The limitations of the present study include reliance on a cross-sectional sample and self-reporting ACEs. This precludes evaluating within-person cognitive decline and increases vulnerability to confounding. However, confounding seems unlikely to account for the largely null results reported here. Several different ACE assessment instruments, reflecting different levels of trauma, are currently in use across the field. The survey used in KHANDLE does not include questions about neglect, physical or sexual abuse, rather it focuses on household dysfunction questions. Our null findings with the nine ACEs assessed in KHANDLE do not rule out the relevance of other ACEs that are more severe. Finally, sample size is an important limitation, although for the overall and race-specific estimates of composite ACE score and cognition associations, CIs were fairly narrow and inconsistent with especially large benefits or harms. KHANDLE is, to our knowledge, the only available community-based study with information on the four largest racial/ethnic groups represented in the USA and rigorous cognitive assessments in older adults. We have reported finely grained results to facilitate future meta-analyses. This study did not directly address dementia because the participants were screened at baseline to be free of dementia. Differences in cognitive performance in late life are relevant for anticipating dementia risk, however, because of the established importance of cognitive reserve. ${ }^{24}{ }^{25}$ Understanding early-life determinants of cognition in older age is important because cognitive function is predictive of myriad health outcomes, including physical health and functional independence as well as dementia. ${ }^{7-9} 26$ Our findings, therefore, if taken at face value, suggest these ACEs may not have major relevance for subsequent dementia risk.

Our results suggest that previously reported findings linking ACEs to cognitive outcomes in late life may be overestimated or may not hold in many communities. These findings should be interpreted cautiously until replicated in additional multiethnic samples. Given the robust evidence of early life experiences overall for cognitive reserve and dementia risk, these results would suggest a focus on other aspects of childhood, such as material deprivation or educational experiences.

\section{Author affiliations}

${ }^{1}$ Department of Epidemiology and Biostatistics, University of California San Francisco, San Francisco, California, USA

${ }^{2}$ Public Health Sciences, University of California Davis, Davis, California, USA

${ }^{3}$ Division of Research, Kaiser Permanente, Oakland, California, USA

${ }^{4}$ Department of Epidemiology, University of California Los Angeles Jonathan and Karin Fielding School of Public Health, Los Angeles, California, USA

Twitter Rachel A Whitmer @Prof_R_Whitmer, Paola Gilsanz @PaolaGilsanz and M Maria Glymour @MariaGlymour

Contributors ALG, EM, SA, DM, RAW, ERM, PG and and MG were involved with the study design, data collection, data analysis and reporting the results. SM and CWE were involved with the data collection, data analysis and reporting the results. All authors revised it critically for important intellectual content. All authors approved the final version of the manuscript and agree to be accountable for all aspect of the work.

Funding This work was supported by the National Institutes of Health grant numbers RF1AG052132, R00AG053410, R01AG066132, T32AG049663 and RF1AG055486.

Competing interests None declared.

Patient consent for publication Not required.

Ethics approval Ethical approval was granted by the Kaiser Permanente Northern California Institutional Review Board (IRB Number: CN-16-2786).

Provenance and peer review Not commissioned; externally peer reviewed.

Data availability statement Data are available on reasonable request. Data may be obtained from a third party and are not publicly available. Deidentified data are available to qualified investigators from the KHANDLE Leadership Committee on approval for the purposes of replicating procedures and results.

Supplemental material This content has been supplied by the author(s). It has not been vetted by BMJ Publishing Group Limited (BMJ) and may not have been peer-reviewed. Any opinions or recommendations discussed are solely those of the author(s) and are not endorsed by BMJ. BMJ disclaims all liability and responsibility arising from any reliance placed on the content. Where the content includes any translated material, BMJ does not warrant the accuracy and reliability of the translations (including but not limited to local regulations, clinical guidelines, terminology, drug names and drug dosages), and is not responsible for any error and/or omissions arising from translation and adaptation or otherwise.

Open access This is an open access article distributed in accordance with the Creative Commons Attribution Non Commercial (CC BY-NC 4.0) license, which permits others to distribute, remix, adapt, build upon this work non-commercially, and license their derivative works on different terms, provided the original work is properly cited, appropriate credit is given, any changes made indicated, and the use is non-commercial. See: http://creativecommons.org/licenses/by-nc/4.0/.

\section{ORCID iDs}

Paola Gilsanz http://orcid.org/0000-0002-7635-381X

M Maria Glymour http://orcid.org/0000-0001-9644-3081

\section{REFERENCES}

1 Felitti VJ, Anda RF, Nordenberg D, et al. Relationship of childhood abuse and household dysfunction to many of the leading causes of death in adults. The adverse childhood experiences (ACE) study. Am J Prev Med 1998;14:245-58.

2 Anda RF, Felitti VJ, Bremner JD, et al. The enduring effects of abuse and related adverse experiences in childhood. A convergence of evidence from neurobiology and epidemiology. Eur Arch Psychiatry Clin Neurosci 2006;256:174-86.

3 Baglivio M, Epps N, Swartz K, et al. The prevalence of adverse childhood experiences (ACE) in the lives of juvenile offenders. Journal of juvenile justice 2014;3:1-23.

4 Barnes LL, Wilson RS, Everson-Rose SA, et al. Effects of early-life adversity on cognitive decline in older African Americans and whites. Neurology 2012;79:2321-7.

5 Korten NCM, Penninx BWJH, Pot AM, et al. Adverse childhood and recent negative life events: contrasting associations with cognitive decline in older persons. $J$ Geriatr Psychiatry Neurol 2014;27:128-38.

6 Ritchie K, Jaussent I, Stewart R, et al. Adverse childhood environment and late-life cognitive functioning: childhood adversity and late-life cognition. Int J Geriatr Psychiatry 2011;26:503-10.

7 Villarejo A, Bermejo-Pareja F, Trincado R, et al. Memory impairment in a simple recall task increases mortality at 10 years in non-demented elderly. Int J Geriatr Psychiatry 2011;26:182-7.

8 Luppa M, Luck T, Weyerer S, et al. Prediction of institutionalization in the elderly. A systematic review. Age Ageing 2010;39:31-8.

9 Wagner DC, Short JL. Longitudinal predictors of self-rated health and mortality in older adults. Prev Chronic Dis 2014;11:E93.

10 Ravona-Springer R, Beeri MS, Goldbourt U. Younger age at crisis following parental death in male children and adolescents is associated with higher risk for dementia at old age. Alzheimer Dis Assoc Disord 2012;26:68-73.

11 Mersky JP, Janczewski CE. Racial and ethnic differences in the prevalence of adverse childhood experiences: findings from a lowincome sample of U.S. women. Child Abuse Negl 2018;76:480-7.

12 Klein NP, Fireman B, Goddard K, et al. Vaccine effectiveness of cellculture relative to egg-based inactivated influenza vaccine during the 2017-18 influenza season. PLoS One 2020;15:e0229279. 
13 Gordon NP, Kaplan GA. Some evidence refuting the HMO "favorable selection" hypothesis: the case of Kaiser Permanente. Adv Health Econ Health Serv Res 1991;12:19-39.

14 Krieger N. Overcoming the absence of socioeconomic data in medical records: validation and application of a census-based methodology. Am J Public Health 1992;82:703-10.

15 Gordon NP. Similarity of the Kaiser Permanente senior member population in northern California to the Non-Kaiser Permanente covered and general population of seniors in northern California: statistics from the 2009 California health interview survey. Kaiser Permanente Northern California Division of Res 2012.

16 Mungas D, Reed BR, Crane PK, et al. Spanish and English neuropsychological assessment scales (SENAS): further development and psychometric characteristics. Psychol Assess 2004;16:347-59.

17 Howard VJ, McClure LA, Glymour MM, et al. Effect of duration and age at exposure to the stroke belt on incident stroke in adulthood. Neurology 2013;80:1655-61.

18 Anda RF, Dong M, Brown DW, et al. The relationship of adverse childhood experiences to a history of premature death of family members. BMC Public Health 2009;9:106.

19 Pechtel P, Pizzagalli DA. Effects of early life stress on cognitive and affective function: an integrated review of human literature. Psychopharmacology 2011;214:55-70.
20 Ivy AS, Rex CS, Chen Y, et al. Hippocampal dysfunction and cognitive impairments provoked by chronic early-life stress involve excessive activation of $\mathrm{CRH}$ receptors. J Neurosci 2010;30:13005-15.

21 Oh DL, Jerman P, Silvério Marques S, et al. Systematic review of pediatric health outcomes associated with childhood adversity. BMC Pediatr 2018;18:83.

22 Howell KH, Miller-Graff LE. Protective factors associated with resilient functioning in young adulthood after childhood exposure to violence. Child Abuse Negl 2014;38:1985-94.

23 Walsh D, McCartney G, Smith M, et al. Relationship between childhood socioeconomic position and adverse childhood experiences (ACEs): a systematic review. J Epidemiol Community Health 2019;73:1087-93.

24 Scarmeas N, Stern Y. Cognitive reserve: implications for diagnosis and prevention of Alzheimer's disease. Curr Neurol Neurosci Rep 2004; 4:374-80.

25 Scarmeas N, Stern Y. Cognitive reserve and lifestyle. J Clin Exp Neuropsychol 2003;25:625-33.

26 Barnes DE, Covinsky KE, Whitmer RA, et al. Predicting risk of dementia in older adults: the late-life dementia risk index. Neurology 2009;73:173-9. 